\title{
Acute transverse myelitis following carpet viper snakebite in Northeast Nigeria
}

\author{
Nura H. Alkali, ${ }^{1}$ Sulaiman T. Sa'ad, ${ }^{2}$ Abubakar Saidu, ${ }^{3}$ Jacob A. Dunga, ${ }^{1}$ Fadimatu K. Sa'ad, \\ Godiya Ishaya ${ }^{1}$ \\ ${ }^{1}$ Department of Medicine, Abubakar Tafawa Balewa University Teaching Hospital Bauchi, Bauchi State; \\ ${ }^{2}$ Department of Radiology, Federal Teaching Hospital Gombe, Gombe State; ${ }^{3}$ Department of Medicine, \\ Federal Teaching Hospital Gombe, Gombe State, Nigeria
}

\begin{abstract}
Snakebite is a major cause of death and disability in developing countries. The common clinical features of snake envenomation include bleeding, acute kidney injury, dilated pupils and muscle paralysis resulting from the deleterious effects of venom toxins on human plasma proteins, cell membranes, muscle fibers and vascular endothelium. Snakes also harbour pathogenic organisms that may infect bite victims during envenomation, but few studies have described this phenomenon. We herein report a rare case of acute transverse myelitis occurring after snakebite in Northeast Nigeria, and discuss the possible role of bacteria and viruses after excluding other potential causes of myelopathy. This knowledge may help improve the care of snakebite victims among internists and emergency physicians.
\end{abstract}

\section{Introduction}

Each year, snakebites affect up to 5.4 million people worldwide to cause approximately 137,880 deaths. ${ }^{1}$ Accurate data are

\footnotetext{
Correspondence: Nura H. Alkali, Department of Medicine, Abubakar Tafawa Balewa University Teaching Hospital, PMB 0117 Bauchi, Bauchi State, Nigeria.

Tel.: +23.48066389950.

E-mail: nalkali@yahoo.com

Key words: Acute transverse myelitis; Myelopathy; Snakebite; Snake venom.

Contributions: the authors contributed equally.

Conflict of interest: the authors declare no potential conflict of interest.

Funding: none.

Received for publication: 20 March 2019

Revision received: 16 November 2019.

Accepted for publication: 16 November 2019.

This work is licensed under a Creative Commons Attribution NonCommercial 4.0 License (CC BY-NC 4.0).

${ }^{C}$ Copyright: the Author(s), 2019

Licensee PAGEPress, Italy

Annals of African Medical Research 2019; 2:70

doi:10.4081/aamr.2019.70
}

scarce in Sub-Saharan Africa, where bite incidence and mortality are often under-reported. ${ }^{1}$ A recent meta-analytic study revealed Nigeria bears the greatest burden in West Africa, with snakebites causing 2,333 deaths and over 4,000 amputations each year. ${ }^{2}$

The clinical features of envenomation mostly result from the effects of venom toxins, which include pro-coagulant enzymes, phospholipases, cytolytic peptidases, neurotoxins and haemorrhagins. $^{3}$

Pro-coagulant enzymes activate human clotting factors to cause bleeding from consumption coagulopathy, while phospholipases $\mathrm{A}_{2}$ damage cell membranes, vascular endothelium and muscle fibers to cause myoglobinuria and kidney injury. ${ }^{3}$ Neurotoxins damage nerve endings and compete with acetylcholine at neuromuscular junction terminals to cause paralysis, which manifests as ptosis, dilated pupils, and limb and bulbar weakness. Other neurologic presentations of snakebite include stroke, seizures, encephalopathy and acute disseminated encephalomyelitis (ADEM), especially with bites from vipers, kraits and cobras. ${ }^{4-6}$

Snakes also harbour pathogenic bacteria and viruses. Studies have reported wild snakes infected with West Nile virus and Eastern Equine Encephalitis virus, ${ }^{7,8}$ while bacteria including Staphylococcus aureus, Escherichia coli, Klebsiella, Pseudomona and Proteus have been isolated from snake oral cavities and infected bite wounds., 9,10 This suggests that envenomation may transmit infectious disease to the bite victim. Indeed, snakebite was associated with acute bacterial meningitis in India. ${ }^{11}$

In this report, we describe Acute Transverse Myelitis (ATM) occurring after snakebite in Northeast Nigeria. To our knowledge, no study has previously associated snakebite with ATM.

\section{Case Report}

An 18-year-old male had a snakebite to his left index finger in June, 2016, while working at a village farm near Potiskum in Yobe State, Northeast Nigeria. He presented to the district hospital two hours later with a swollen hand and forearm along with the killed snake, which health-workers identified as the West African carpet viper Echis occelatus.

He had a positive 20-minute whole blood clotting test and received $40 \mathrm{~mL}$ of polyvalent snake antivenom (Vins Bioproducts, India) mixed in intravenous (IV) normal saline. Swelling subsided by the next day, and he was discharged home with a normal clotting profile and no signs of systemic envenomation. Three days later, he developed fever, neck pain, tingling over the arms, a progressive weakness of all four limbs, and urinary retention.

He returned to the same hospital, and was diagnosed with "tetanus". He received intramuscular (IM) tetanus toxoid $0.5 \mathrm{~mL}$ stat; IV anti-tetanus serum 10,000 units stat; IV diazepam $10 \mathrm{mg}$ 
given slowly; and IV crystalline penicillin 1.5 million units given 6-hourly. He was then referred to our tertiary hospital when he failed to improve by the second day.

Further history revealed he had not experienced spasms, trismus or opisthotonus suggestive of tetanus. Examination showed he had fever (temperature, $38.4^{\circ} \mathrm{C}$ ) and healed fang marks on the left index finger. He was conscious and alert, with normal cranial nerves, no signs of meningeal irritation and no muscle spasms.

There was global hypotonia, increased deep tendon reflexes, bilateral extensor plantar responses and power of grade $0 / 5$ (Medical Research Council) in all four limbs. All modalities of sensation were absent from the feet up to C8/T1 dermatomes on both sides, but normal on the face and neck. We diagnosed acute transverse myelitis to keep in view haematomyelia from consumption coagulopathy.

Investigations, which included serum electrolytes, urea, creatinine, calcium, albumin, liver enzymes, fasting blood glucose, full blood counts and erythrocyte sedimentation rate were all normal. Blood film showed no malaria parasites, haemoglobin genotype was AA and two blood cultures were negative. Serial international normalized ratios (INR) were 1.2 to 1.5 times control values. Serum rheumatoid factor, antinuclear antibodies and viral screening for HIV and hepatitis B and C infections were all negative.

Chest $\mathrm{x}$-ray and brain magnetic resonance imaging (MRI) were normal. However, cervical spine MRI in sagittal view showed a 10 $\mathrm{mm}$-long lesion which was hyperintense on T2-weighted sequence (T2W; Figure 1) and hypointense on T1W sequence. Post-contrast imaging showed enhancement with gadolinium. The axial T2W image showed a cross-sectional spinal cord lesion consistent with transverse myelitis.

Lumbar puncture revealed normal cerebrospinal fluid (CSF) protein, glucose and cell counts with negative Gram stain and no bacterial growth. CSF Ziel-Nielsen and PCR for Mycobacterium tuberculosis were also negative. Urine microscopy showed pus cells while culture yielded growth of Staphylococcus aureus sensitive to gentamycin and levofloxacin.

He received IV methylprednisolone $250 \mathrm{mg}$ diluted in normal saline once daily for five days, followed with oral prednisolone 30 $\mathrm{mg}$ daily for five days, which was then tapered off. He also received IM gentamycin $80 \mathrm{mg}$ thrice daily for seven days and subcutaneous enoxaparin $20 \mathrm{mg}$ daily for two months. He had daily physiotherapy until his discharge three months later, when he was continent of urine but still bed-ridden. At his last out-patient clinic visit six months after discharge, he had power of grades $4 / 5$ in each upper limb and $2 / 5$ to $3 / 5$ in the lower limbs, with bilateral hypoesthesia below T1 levels.

A sign informed consent to publish this report was obtained from the patient's father before discharge from hospital.

\section{Discussion}

This snakebite patient initially presented with features of mild envenomation, which subsided with polyvalent snake antivenom administered at the local hospital. He went home the next day but returned three days later with fever, quadriparesis, urinary incontinence and a C8/T1 sensory level suggestive of myelopathy. Spinal MRI performed seven days after the snakebite revealed findings consistent with ATM.

ATM is characterized by segmental inflammation of the spinal cord which results in axonal demyelination and necrosis. ${ }^{12}$ Most cases are idiopathic in nature, but ATM is also associated with multiple sclerosis, neuromyelitis optica, sarcoidosis, systemic lupus erythematous (SLE), antiphospholipid syndrome, Sjogren syndrome, Behcet's disease and several pathogenic infections. ${ }^{12}$

Multiple sclerosis is rare in Sub-Saharan Africa, and commonly presents with optic neuritis and demyelinating lesions on brain MRI, thus making it unlikely in this patient. He also lacked the fever, skin rash, arthritis, anemia and raised ESR suggestive of multisystem disease such as SLE, rheumatoid arthritis, Sjogren's disease or antiphospholipid syndrome. The negative serum rheumatoid factor and ANA also made autoimmune disease unlikely.

Sarcoidosis often presents with erythema nodosum, lymphadenopathy, arthritis, lung involvement, parotid swelling or other manifestations, none of which was observed in this case. Serum calcium was also normal, though we couldn't assay serum Angiotensin Converting Enzyme that is sometimes raised in sarcoidosis. ${ }^{12}$ Behcet's disease was also unlikely since he lacked the mouth ulcers, genital ulcers, skin lesions and uveitis commonly seen in patients. ${ }^{13}$ Although ATM may be the initial presentation of Behcet's disease, a concomitant uveitis is often present.

Viruses may cause myelitis either in isolation or as part of an encephalomyelitis. Viral causes of ATM include HIV, herpes simplex virus, cytomegalovirus, Epstein-Barr virus and dengue virus, whereas Zika virus and Eastern Equine Encephalitis virus are often associated with encephalomyelitis. ${ }^{12}$ Our patient was negative for HIV infection; however, we couldn't screen for other viral infections besides hepatitis $\mathrm{B}$ and $\mathrm{C}$.

Bacteria associated with ATM include Klebsiella, Mycoplasma, Staphylococcus aureus, Burkholderia pseudomallei, Borrelia burgdorferi, Orientia tsutsugamushi and Mycobacterium tuberculosis. ${ }^{12}$ In these cases, myelitis is believed to be immunemediated, though direct intramedullary infections have also been reported.

In the case of our patient, urine culture yielded growth of Staphylococcus aureus but blood and CSF cultures were negative. It remained unclear whether antibiotic therapy at the referring hos-

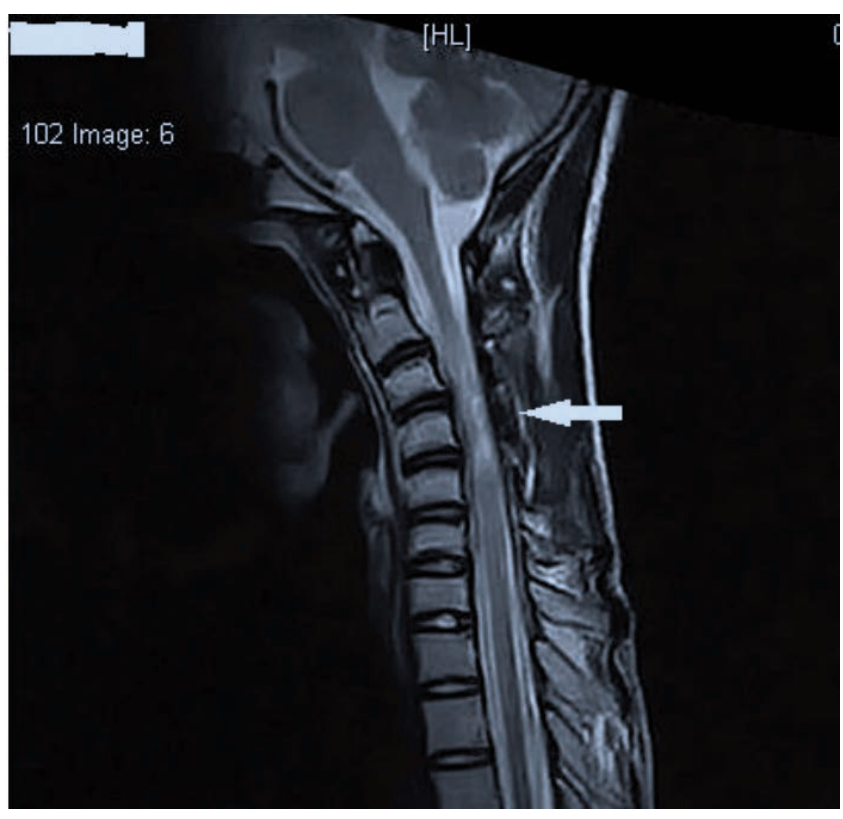

Figure 1. A spinal magnetic resonance image in T2-weighted sequence showing a $10 \mathrm{~mm}$-long hyperintense signal consistent with transverse myelitis (arrow). 
pital affected the blood and CSF cultures or the urine result was due to contaminated specimen. We favoured staphylococcal infection in view of the fever and urine pus at presentation that both resolved with antibiotic therapy.

At least six reports have described ATM occurring in patients with Staphylococcus aureus bacteremia associated with pneumonia, osteomyelitis and brain abscess. ${ }^{14}$ As in our patient, most of the reported cases involved the cervical spinal cord, and one patient with negative blood cultures had bronchoalveolar lavage which cultured methicillin-resistant Staphylococcus aureus. ${ }^{15}$

The source of staphylococcal infection in our patient remained unclear. We excluded urethral catherization since both fever and myelitis developed before the procedure, but we could not determine whether infection was transmitted from snake or was the result of a contaminated bite wound.

\section{Conclusions}

ATM is a rare manifestation of snake envenomation. Clinicians may consider the diagnosis in snakebite patients presenting with features of myelopathy. Spinal MRI where available will help to support the diagnosis and exclude other forms of myelopathy such as hematomyelia. Early treatment with steroids and antibiotics may help improve neurologic outcome.

\section{References}

1. World Health Organization: Snake Envenoming, published April 8, 2019. Accessed November 15, 2019 at https://www.who.int/news-room/fact-sheets/detail/snakebiteenvenoming.

2. Habib AG, Kuznik A, Hamza M, et al. Snakebite is underappreciated: Appraisal of burden from West Africa. PLoS Negl Trop Dis 2015;9:e0004088.

3. Chisale M. Snake and snakebites - Part 2. World Health
Organization Pharmaceuticals Newsletter 2008; 5(1).

4. Margekar SL, Gaharwar R, Jayant SS, et al. Encephalopathy: An unusual neurological manifestation following snakebite. Ind J Clin Pract 2013;24:556-8.

5. Paul G, Paul BS, Puri S. Snakebite and stroke: Our experience of two cases. Ind J Crit Care Med 2014;18:257-8.

6. Singh RB, Kumar A. Neuronal cell damage and stroke in a female following snakebite. JSM Cell 2014;2:1009.

7. Dahlin CR, Hughes DF, Meshaka Jr. WE, et al. Wild snakes harbour West Nile Virus. One Health 2016:136-8.

8. Graham SP, Hassan HK, Chapman T, et al. Serosurveillance of Eastern Equine Encephalitis Virus in amphibians and reptiles from Alabama, USA. Am J Trop Med Hyg 2012;86:540-4.

9. Ghosh T, Biswas MK, Roy P, Guin C. Short review of different microflora from the oral cavity of snakes. Uttar Pradesh J Zool 2017;37:30-4.

10. Chen CM, Wu KG, Chen CJ, Wang CM. Bacterial infection in association with snakebite: A 10-year experience in a northern Taiwan medical center. J Microbiol Immunol Infect 2011;44:456-60.

11. Aundhakar SC, Bharadiya AA, Panpalia GN, Bhalla DG. Snakebite complicated by bacterial meningitis. Int J Med Toxicol Forensic Med 2013;3:34-7.

12. West TW. Transverse myelitis: A review of the presentation, diagnosis, and initial management. Discov Med 2013;16:16777.

13. Bitik B, Ucar M, Tezcan ME, et al. Transverse myelitis in Behcet's disease: a series of four cases and review of the literature. Clin Exper Rheumatol 2013;31:S20-4.

14. Karakonstantis S, Galani D, Maragou S, et al. A rare case of acute transverse myelitis associated with Staphylococcus aureus bacteremia and osteomyelitis. Spinal Cord Ser Cases 2017;3:17029.

15. Kalkan G, Karaoglu A, Vurucu S, et al. Methicillin-resistant Staphylococcus aureus pneumonia accompanied with transverse myelitis. Eur J Gen Med 2012;9:203-4. 